\title{
The Deployment of Sensor Nodes Based on the Internet of Things for Intelligent Agricultural Monitoring
}

\author{
Zeping $\mathrm{Mu}^{*}$ \\ School of Software Engineering, Chongqing College of Electronic Engineering, Chongqing, China \\ ${ }^{*}$ Corresponding author
}

\begin{abstract}
Sensor networks can be applied to the deployment of intelligent monitoring of ecological environment with the fewest sensor nodes. First, determine the factors under monitoring and the critical points of relevant factors. Next, build the optimized mathematical monitoring model in terms of the monitored factors and critical points. Last, solve the mathematical model and find the critical points with the most properties as the preselected point.
\end{abstract}

Keywords-internet of things (IoT); agricultural monitoring; sensor nodes; node deployment

\section{INTRODUCTION}

As an important part of new generation of information technology, the Internet of Things (IoT) was officially listed as one of the seven major emerging strategic industries, and was documented in the government work report, consequently, its application domain is expanding continuously. Beside, The National Development and Reform Commission (NDRC) made IoT-specific researches ${ }^{[1]}$, and the Ministry of Industry and Information Technology (MIIT) deployed "The Internet of Things second five development plan" ${ }^{2,3]}$. Meanwhile, China will continuously strive to foster strategic merging industries, and develop the idea of "cloud computing IoT" ${ }^{[2,3]}$. These government initiatives can have certain scientific significance and application prospect for researches into IoT application to citrus monitoring. Node deployment can be of great significance to researches into the prerequisites of IoT application to citrus monitoring, and into sensor node deployment.

The application of wireless sensor network (WSN) usually involves node deployment. This issue is quite crucial in widecovering environmental monitoring sensor networks that consist of a large amount of nodes. Environmental monitoring applications can be divided into two categories, anomaly detection and normal continuous sampling monitoring.

In the anomaly detection like earthquake early warning or forest fire protection, the monitoring of sensor networks is to detect the specific place and time of anomaly occurrence.

In the normal continuous sampling monitoring like water quality monitoring, ecological monitoring, also including orchard environment monitoring studied in this article, the monitoring of sensor networks is to continuously sample the environment parameters at the nodes, and estimate the data of non-node position by the interpolation method in statistics to analyze the continuous changes of monitored data in space and time.

In the node deployment of anomaly detection, many scholars at home and abroad currently locate the research background at anomaly detection or target tracking, and tend to consider the deployment issue from the two aspects connectivity and coverage. Connectivity is the property of transferring the information collected by nodes precisely to base stations, generally within the node communication range. Coverage is to ensure that nodes can cover the whole working region, and collect target information completely and accurately within the node sensing range in most occasions. The latter can investigate the relationship between node communication range and node sensing range, before providing the optimization theory about node deployment, best satisfying connectivity and coverage requirements.

As for the node deployment of normal continuous sampling monitoring, some node deployment theories about anomaly detection cannot be fully applicable to the category like orchard environmental monitoring. However, node deployment issue at present is only mentioned in few literatures on farmland environmental monitoring and other normal continuous sampling monitoring. For instance, Alberto (2007) deploys nodes in the regular grid, in his modeling simulation experiment of farmland information based on WSN. Also, Robert (2007) deploys one monitoring node in each irrigation management zone in his irrigation control study based on WSN Nevertheless, there is lack of systematic and practical theoretical research into the deployment method of sensor network nodes in the normal continuous sampling.

In the traditional method of ecological environment monitoring, it can be greatly subjective and arbitrary to select monitoring nodes, and may lack relevant quantitative mathematical model, thus it is probably hard in practice to balance the monitoring effect and construction costs. The cost of ecological environment monitoring is mainly from facility monitoring. On the premise of qualified monitoring performance, reducing the monitoring nodes can be the best way to cut costs. This way reduces not only facility costs but also the energy consumption in the future use. Therefore, the 
author proposes one method to deploy with the fewest sensor nodes the intelligent monitoring of ecological environment.

\section{NODE DEPLOYMENT OptiMIZATION AlgORITHM}

Node deployment optimization algorithm has two main goals: One is to determine the fewest monitoring nodes on the premise of completing the monitoring to all the indexes. The other is under the control of monitoring node quantities, setting the position approximate to the attribute mean of distribution as the deployment point of monitoring nodes, so as to obtain the general and representative results.

Adopting the presetting system, the algorithm establishes a parameter for each index, and expresses the possible distributions with Field[a][b] in terms of land status. Field represents monitoring indexes; [a] land status, the a-value is natural number; and [b] possible distributions, b-value is likewise natural number. Similarly, Isoheight[a][b] represents the isoheight status, Water-area[a][b] water status, Fertilizer[a][b] fertilizer status, Pollution[a][b] pollution status, and landscape[a][b] landscape status.

On the whole distribution, the ultimate goal is to save system costs and realize the best monitoring results with the deployment of the fewest monitoring nodes. Therefore, it is assumed that the same position on the distribution with the most monitoring effects is set as the final choice, as shown formula (1).

$$
\begin{aligned}
& \text { Point }_{[x][y]}=\max \left(\text { Field }_{[a][b][x][y]}+\text { Isoheight }_{[a][b][x][y]}+\text { Water }-\operatorname{area}_{[a][b][x][y]}\right. \\
& + \text { Fertilizer }_{[a][b][x][y]}+\text { Pollution }_{[a][b][x][y]}+\text { landscape }_{[a][b][x][y]} \text { ) }
\end{aligned}
$$

In the formula 1, the evaluations of Isoheight, Water-area, Fertilizer, Pollution and landscape are shown below from formula 2 to formula 7 , where the $[\mathrm{x}]$ and $[\mathrm{y}]$ represent the node coordinates.

$$
\begin{aligned}
& \text { Field } d_{[a][b]}=\left\{\begin{array}{cc}
1 & \in \text { field } \\
0 & \text { other }
\end{array}\right\} \\
& \text { Isoheight }_{[\mathrm{a}][\mathrm{b}]}=\left\{\begin{array}{cc}
1 & \in \text { Isoheight } \\
0 & \text { other }
\end{array}\right\} \\
& \text { Water }-\operatorname{area}_{[\mathrm{a}] \mathrm{b}]}=\left\{\begin{array}{cc}
1 & \in \text { Water }- \text { area件 } \\
0 & \text { other }
\end{array}\right\} \\
& \text { Fertilizer }_{[\mathrm{a}][\mathrm{b}]}=\left\{\begin{array}{cc}
1 & \in \text { Fertilizer } \\
0 & \text { other }
\end{array}\right\}
\end{aligned}
$$

$$
\begin{aligned}
& \text { Pollution }_{[a][b]}=\left\{\begin{array}{cc}
1 & \in \text { Pollution } \\
0 & \text { other }
\end{array}\right\} \\
& \text { landscape }_{[\mathrm{a}][\mathrm{b}]}=\left\{\begin{array}{cc}
1 & \in \text { landscape } \\
0 & \text { other }
\end{array}\right\}
\end{aligned}
$$

After several loops from formula (2) to formula (7), all the positions are transformed into a Point 6 , which is shown in formula 8:

In the formula (8), [v] is an occurrence. For example, Field is specific appropriate parameter, and Isoheight is altitude value. Meanwhile, store Point $t_{[x][y]}$ into an array from large to small, and when the values of Point $t_{[\mathrm{x}][\mathrm{y}]}$ are the same, its storing sequence is determine by formula (9).

$$
\text { point }=\left[\begin{array}{c}
\text { field }_{[a][b][x][y][v]} \\
\text { Isoheight } \\
\text { Water }- \text { area }_{[a][b][x][y][v][x][y][v]} \\
\text { Fertilizer } \\
\text { Pollution }_{[a][b][x][y][[v][x][y][v]} \\
\text { Landscape }_{[a][b][x][y][v]}
\end{array}\right]
$$

$$
h=\max \left(n_{1}, n_{2}, \ldots \ldots\right)
$$

And its calculation of ni is shown in formula (10):

$$
\begin{aligned}
& n_{i}=\frac{\mid \text { field }_{v}-\operatorname{avg}\left(\text { field }_{[a]}\right) \mid}{\operatorname{avg}\left(\text { field }_{[a]}\right)}+ \\
& \frac{\mid \text { Isoheight }_{v}-\operatorname{avg}\left(\text { Isoheight }_{[a]}\right) \mid}{\operatorname{avg}\left(\text { Isoheight }_{[a]}\right)}+\ldots \ldots
\end{aligned}
$$

After the calculation in the formula (9) and (10), a Point in a ranking array can be obtained. From the last one in the array, one Point matrix can be deleted if it has the same property as the previous one.

\section{The ApPlicAtion OF NODE DePlOyMENT OPTIMIZATION ALGORITHM}

\section{A. Deployment Background}

The overall optimization algorithm is applied to the modern sightseeing eco-agricultural zone in the western China, in which there are citrus planting area of $14800 \mathrm{mu}, 580,000$ planting citrus, $103 \mathrm{~km}$ road in the zone, mechanization irrigation area $500 \mathrm{mu}$ and drip irrigation area $4600 \mathrm{mu}$. There 
are selected $3000 \mathrm{mu}$ monitoring area and 10,000 planting citrus in our experiment,as shown in Figure 1. The land status, isoheight, the distance from water area, fertilizer water stations, and industrial area, power supply conditions, signal transmission distance and other factors are mainly considered as preselected points in the whole program. In addition, soil moisture and temperature, air humidity and temperature, illumination, wind speed and direction, rainfall and other information of citrus production environment are under the supervision. The deployment of sensor nodes after optimization is presented in the Figure 2.

In the Figure 2, the No.1 node is responsible for monitoring the farmland while the No.6 node is for dry land conditions. No. 2, 3, 7 and 8 aim at the mountainous conditions, also, the No. 2 node is for landscape, fertilizer and high-altitude region. No. 1 , 4, 5, 9 are for low-altitude regions. No. 3, 7, 8 are for averagealtitude regions; No. 3 and No. 9 mainly monitor the impact of industries on this region. No. 4 is for the place with rich water resource, while No. 3, 6, 7, 8, 9 are for those relatively short of water; No.5 node is for greenhouse monitoring; No.7 and No.8 are for the long-distance transmission of information.

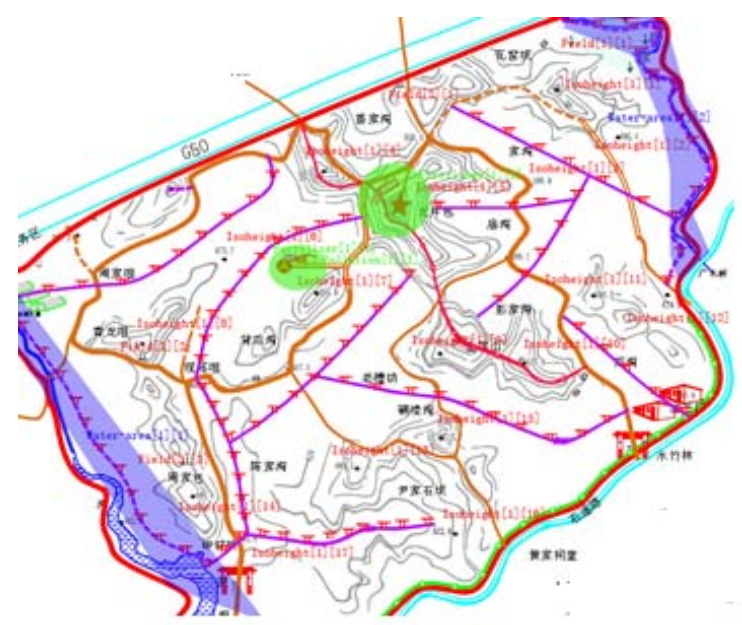

FIGURE I. MONITORING GARDEN

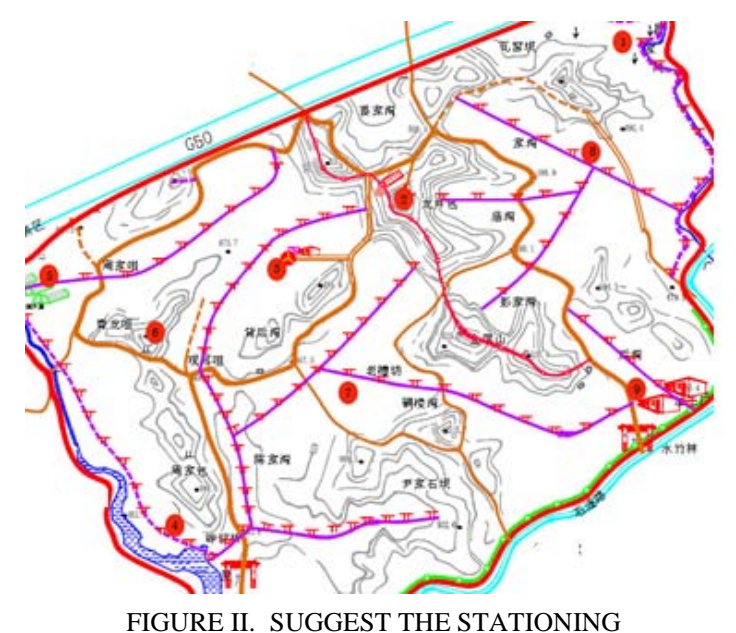

FIGURE II. SUGGEST THE STATIONING

\section{B. The Cost Comparison of Various Deployment Methods}

The information monitoring costs of citrus parks consists of node material costs and the communication costs of renting optical fibers, and both are determined by the number of nodes, while in regular grid deployment method, it depends on monitoring area and node coverage radius. Supposing set the monitoring area as $\mathrm{S}$ and node coverage radius as $\mathrm{r}$, so the number of needed nodes expressed as $\mathrm{n}$, can be calculated by formula (11).

$$
n=S / 2 r * 2 r=S / 4 r^{2}
$$

The number of node deployment correlates with regional property as well as regional distribution of the chosen property. The best case is there can be one critical point possessed with all the properties, so the number of monitoring nodes is one; while the worst case is that each critical point has only one and different property, so the number of monitoring nodes by OASNDFA algorithm is $\mathrm{n}$, approximate to the result in grid deployment method. However, this case can seldom exist in practice. For the convenience of comparison, taking the monitoring zone of $3000 \mathrm{mu}$ mentioned in the article as the object, compare its profits in grid deployment method without using the information and in OASNDFA algorithm, and the results are presented, as shown Figure 3.



In the Figure 3, "1" represents the expenditure comparison, " 2 " is the total assets, and " 3 " is the balance of profits minus costs. It can be concluded from the chart that grid deployment method probably applies to precise crops on the small scale while the algorithm proposed in this article can be widely applicable, especially to large-scale cultivation.

\section{CONCLUSION}

In this article, the author proposes one method to deploy with the fewest sensor nodes the intelligent monitoring of ecological environment. It first determines the factors needed to be monitored and the critical points of relevant factors. Next, it can build the optimized mathematical monitoring model in 
terms of the monitored factors and critical points. Then the mathematical model would be solved to determine the critical points with the most properties as the preselected point. Finally, it can be tested on spot research that the deployment can reach the results as in the grid deployment monitoring, and the use nodes far fewer than those in the grid algorithm.

\section{ACKNOWLEDGMENT}

This work was supported by the National High-Tech R\&D Program (863 Program 2015AA01A705), Chongqing Education Science Project [kj1402905], Chongqing Twelfth Five Year plan educational projects [2013-ZJ-077], Ministry of Education and Mobile Research Fund Project of China [MCM20130661]

\section{REFERENCES}

[1] National Development and Reform Commission made IoT-specific researches. http://www.sdpc.gov.cn/zcfb/zcfbtz/2012tz/t20120518 4802 81. htm. 2012.5.15

[2] Ministry of Industry and Information Technology (MIIT) deployed "The Internet of Things second five development plan”. http://www.gov.cn /zwgk/2012-02/14 /content_2065999.htm,2012.2

[3] 2012 the government work report, "the Internet of things cloud computing named support”. http://www.gbds.com.cn/gdzx/rdld/wlw/20 1203/t20120307_1257526.shtml. 2012-03-07

[4] Alberto Camilli, Carlos Eduardo Cugnasca, Antonio M Saraiva,et al. From wirless sensors to field mapping: Anatomy of an application for precision agriculture[J]. Computers and Electronics in Agriculture. 2007, 58(1): $25-36$ 